\title{
Expanding a dynamic flux balance model of yeast fermentation to genome-scale
}

\author{
Felipe A Vargas, Francisco Pizarro, J Ricardo Pérez-Correa and Eduardo Agosin ${ }^{*}$
}

\begin{abstract}
Background: Yeast is considered to be a workhorse of the biotechnology industry for the production of many value-added chemicals, alcoholic beverages and biofuels. Optimization of the fermentation is a challenging task that greatly benefits from dynamic models able to accurately describe and predict the fermentation profile and resulting products under different genetic and environmental conditions. In this article, we developed and validated a genome-scale dynamic flux balance model, using experimentally determined kinetic constraints.

Results: Appropriate equations for maintenance, biomass composition, anaerobic metabolism and nutrient uptake are key to improve model performance, especially for predicting glycerol and ethanol synthesis. Prediction profiles of synthesis and consumption of the main metabolites involved in alcoholic fermentation closely agreed with experimental data obtained from numerous lab and industrial fermentations under different environmental conditions. Finally, fermentation simulations of genetically engineered yeasts closely reproduced previously reported experimental results regarding final concentrations of the main fermentation products such as ethanol and glycerol.
\end{abstract}

Conclusion: A useful tool to describe, understand and predict metabolite production in batch yeast cultures was developed. The resulting model, if used wisely, could help to search for new metabolic engineering strategies to manage ethanol content in batch fermentations.

\section{Background}

Management of ethanol yields is emerging as one of the most relevant challenges for biotechnology, including both ethanol maximization (i.e. bioethanol and distilled beverages industries) and reduction/minimization (i.e. wine, bakery and commodities industries). Significant advances have been made in modeling ethanol fermentations [1-5], although steady-state, gene-modification strategies have resulted in varying degrees of success (for review see [6-8]) mainly due to growth impairment. In turn, dynamic models are able to describe and predict batch fermentations' time-courses better, since different metabolic stages are considered.

A widely used modeling approach to predict cell behavior beyond calibration data is Flux Balance Analysis (FBA), which represents cell biochemical networks as a set of underdetermined constrained mass-balances. In

\footnotetext{
* Correspondence: Agosin@ing.puc.cl

Department of Chemical and Bioprocess Engineering, School of Engineering, Pontificia Universidad Católica de Chile, Casilla 306 - Correo 22, Santiago
} CHILE

(c) 2011 Vargas et al; licensee BioMed Central Ltd. This is an Open Access article distributed under the terms of the Creative Commons Attribution License (http://creativecommons.org/licenses/by/2.0), which permits unrestricted use, distribution, and reproduction in any medium, provided the original work is properly cited. this framework, linear programming is applied to generate a flux distribution that optimizes a given objective function, subject to flux balance equations and constraints. Objective functions commonly used are maximization of ATP production $[9,10]$, maximization of biomass formation [11-13], minimization of metabolic adjustment (MoMa) [14] or minimization of ATP consumption $[15,16]$. So far, growth maximization has been the most extensively used approach to describe the physiology during growth.

Applying FBA in large-scale metabolic reconstructions, termed Genome-Scale metabolic models (GSMM), has allowed establishing a direct relationship between genetic data and metabolic activity fluxes. These models have been shown to be very useful in predicting the physiological behavior of a microorganism under different genetic and environmental disturbances, i.e. growth rate and product secretion patterns [11,13,17-19]. GSMM can help to speed up the design of cells with improved and desired properties, providing metabolic engineering targets that are experimentally testable
C Biomed Central 
[20-22]. For example, bacterial genome-scale models have been used to design strains that overproduce lycopene [23], lactate [24], succinate [25], 1,3-propanediol [26], hydrogen [20], L-valine [27] and L-threonine [28]. In turn, genome-scale yeast models have been mainly applied to design ethanol overproducing strains [22,29-31]; nevertheless, design of ethanol underproducing strains have been overlooked, a key issue in wine industry today [5,32-34].

Many computational tools for identifying strain modifications leading to targeted overproductions have been described in the literature. One of the earliest efforts was the OptKnock [26] procedure that proposed gene knockouts leading to targeted overproductions. Later, OptReg [35] expanded OptKnock to consider not only knockouts but also overexpressions and down regulations. In addition, OptStrain [20] allowed for knock-ins of non-native genes to enable production of desired biochemicals. Evolutionary search procedures for solving the resulting combinatorial optimization problems were explored in OptGene [36]. Recently, OptForce [21] was used to identify flux manipulation leading to targeted overproductions. However, strain optimization requires taking into account the whole bioprocess, i.e. growth as well as non-growth periods, such as lag, log and steadystate phases. Furthermore, strain optimization needs microbial metabolic modeling expanded upon constraint-based FBA that incorporates experimental validation [37], which has not always been considered in GSMM development. Including these issues will improve the capability of the models to predict the impact of several environmental and genomic alterations on the course of batch fermentations.

Several yeast genome-scale metabolic models have been described so far: iFF708, iND750, iLL672, iIN800, iMM904 and Yeast $4.0[18,19,38-41]$. Despite their advantages, all of them can only simulate time-invariant extracellular conditions consistent with continuous culture, but are not able to reproduce features of the microbial growth process. Dynamic FBA models are increasingly applied to simulate bacteria [42-45], yeast $[15,46,47]$, plant [48] and animal $[49,50]$ growth under several conditions $[51,52]$. However, the application of dynamic genome-scale FBA models for yeast $[29,30]$ and bacteria [52-54] has been barely explored, and only one bacterial dynamic GSMM model has been experimentally validated.

Over the past few years, our group has been developing a dynamic FBA model (DFBA) to represent anaerobic $S$. cerevisiae batch fermentations [15,47]. This model, comprising a reduced stoichiometric network (39 metabolic reactions), accurately described fermentation profiles under different environmental conditions. However, the lack of detailed metabolic description makes it unsuitable for metabolic engineering studies. To overcome this limitation, we expanded our previous DFBA model of $S$. cerevisiae metabolism $[15,47]$ to genomescale. This model included kinetic expressions to dynamically constrain the uptake of nutrients, biomass and maintenance. In addition, key improvements related to anaerobic metabolism were identified. Furthermore, we carried out a comprehensive validation using experimental profiles of batch fermentations and final concentration of metabolic products under genetic/environmental disturbances. To the best of our knowledge, we report here the first experimentally validated GS-DFBA for $S$. cerevisiae.

\section{Methods}

\section{Model formulation}

The current version of our genome-scale dynamic-fluxbalance-analysis model (GS-DFBA) consists of four interacting blocks, which are solved sequentially (Figure 1). First, initial conditions and fixed constraints are specified (block 0). Next, dynamic constraints which depend on metabolite concentrations in the medium are established (block 1). A linear programming (LP) problem is then solved (block 2) to compute the consumption and production rates of extracellular metabolites. Finally, these production rates are transferred to an ODE solver (block 3 ) that integrates the respective bioreactor mass balances during intervals of 30 minutes (keeping extracellular rates constant), providing accurate results without losing fermentation details [15,47]. A sensitivity analysis showed small variations when this time interval is changed (Additional file 1). The procedure iterates between blocks 1, 2 and 3 until the sugar is consumed or an unfeasible condition is found.

Typical initial conditions that must be defined a priori are temperature, and the concentration of nitrogencompounds, sugar-compounds, extracellular metabolites and biomass. The LP is bounded by fixed and dynamic constraints. Fixed constraints include carbon/nitrogen limiting medium, oxygen presence or absence, biomass equation, and genetic background. Dynamic constraints include sugar/nitrogen uptake kinetics, maintenance and carbohydrate accumulation. Following this procedure, it takes $43 \mathrm{~s}$ of CPU time to simulate a normal alcoholic fermentation (200 g/L of sugars and $300 \mathrm{mg} / \mathrm{L}$ of assimilable nitrogen) in a Core2Duo $2.66 \mathrm{GHz}$ processor. The features of each block are detailed below (Figure 1).

\section{Linear programming}

A standard FBA model $[13,55,56]$ comprises an underdetermined metabolic network of $\mathrm{s}$ molecular species and $v$ reactions, which is represented by a $v \times \mathrm{s}$ stoichiometric matrix $(\mathrm{T})$. To solve the intracellular fluxes as well as the consumption and production rates of metabolites in the cell, an LP is defined (eq. 1), where 


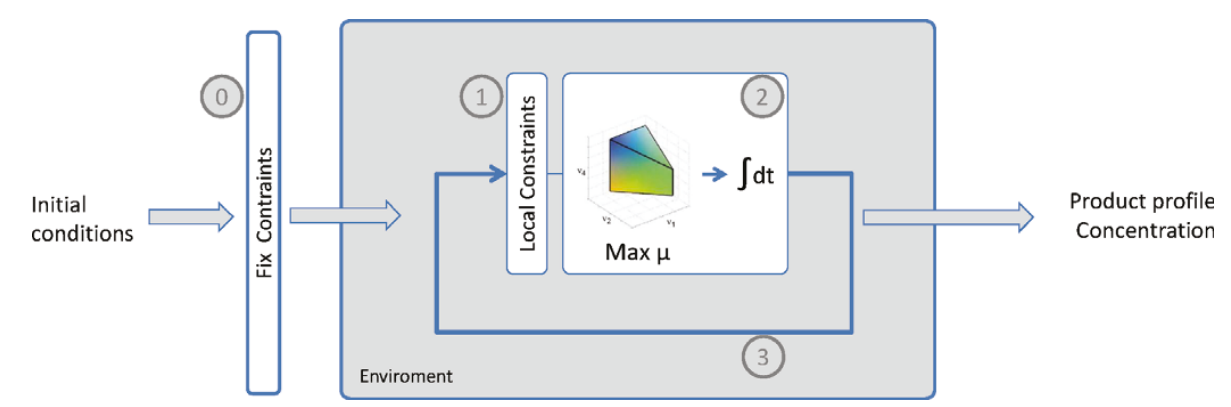

Figure 1 Resolution algorithm of idFV715 model. The model is based on an iterative optimization of an under determined matrix, using LINDO optimization software (LINDO system): 0) Fixed constraints to be used throughout fermentation are defined, such as genetic background or nutritional requirements; 1) Dynamic constraints are defined as bounds fluxes set to by intracellular extracellular conditions; 2) LP solves the metabolic flux distribution, as well as the consumption and production rates, at 30 min intervals; 3) The resulting rates are used as inputs for the differential equations solved using a variable step integration routine.

an objective function for cell metabolism $v_{j}$ should be specified. Following common practice, maximization of growth rate was used to mimic growth in exponential phase [11-13]; however, when the limiting nutrient is exhausted, minimization of ATP consumption by maintenance was applied $[15,16]$.

$$
\begin{aligned}
\operatorname{Min}\left\{-v_{j}\right\} \text { Subject to } & T^{\prime} \cdot v=0 \\
& v_{i}^{L B} \leq v_{i} \leq v_{i}^{U B}
\end{aligned}
$$

where $v$ is the vector of metabolic fluxes, and $v_{i}^{\mathrm{LB}}$ and $\nu_{i}^{\mathrm{UB}}$ are dynamic lower and upper bounds of the flux $i$, respectively. All LP calculations were carried out within MATLAB (The MathWorks Inc.) using Lindo optimization package (Lindo Systems Inc.).

To reduce the impact of multiple optimal solutions that normally appear in FBA [57], for metabolic engineering approaches we carried out a bi-level optimization procedure [29], solving first the LP for maximum biomass, and then fixing the biomass at this maximum value and solving the LP again to optimize a second objective (i.e. maximizing/minimizing ethanol).

Up until now, several yeast genome-scale model databases have been published: iFF708 [18], iND750 [19], iLL672 [40], iIN800 [39], iMM904 [38] and Yeast 4.0 [41]; each one adding more details to the metabolic network. However, additional information regarding compartmentalization, cycles and expansion of previously lumped reactions has not necessarily led to improved accuracy and better predictions.

The first published genome-scale model, iFF708, was the simplest and has good predictive performance. Therefore, The GS-DFBA model described here was developed using an updated version of the iFF708 stoichiometric matrix [18] coupled with our previously developed algorithms of DFBA (Figure 1) [15,47]. The resulting model, idFV715, where "d" stands for dynamic and " $\mathrm{i}$ " for in silico, consists of 715 structural ORF's that catalyze 705 distinct biochemical reactions and 145 putative reactions that have not been associated with any gene yet, comprising 590 metabolites and 1181 metabolic reactions in total. Compared with iFF708, the idFV715 model includes a more detailed description of flavor production related to acetoin and butanediol syntheses.

\section{Dynamic mass balance}

During microbial growth, the environmental conditions are changing constantly due to the consumption of nutrients and secretion of metabolites. These changes are modeled by the following set of ordinary differential equations:

$$
\frac{d M_{E X}}{d t}=V_{E X} \cdot X_{V}
$$

Where $M_{E X}$ is the vector of concentrations of extracellular metabolites in the medium, $V_{E X}$ is the vector of the respective specific consumption and production rates, and $X_{V}$ is the concentration of viable biomass in the medium.

\section{Fixed constraints}

These include all constraints that remain constant during the whole fermentation, such as genetic background (deletions, insertions and overexpressions), carbon/nitrogen limiting medium that modify the biomass equation, and lack of oxygen that modify oxygen-related reactions.

As yeast responds differently to aerobic or anaerobic conditions [58-60] we described the S. cerevisiae physiology under these conditions as closely as possible. The expression of approximately one hundred genes is regulated by oxygen availability $[61,62]$, affecting mainly the cell redox state [63]. This, in turn, affects ATP, biomass and product syntheses. For instance, approximately eight times less ATP and ten times less biomass are produced under anaerobic conditions [64,65].

In our approach, we bounded to zero the oxygen uptake-related flux. In addition, we included a known 
transcriptional regulation, i.e. during anaerobic fermentation the TCA cycle has been shown to be broken at the level of succinyl CoA synthetase, acting as two separate branches - the "oxidative branch" and the "reductive branch." This behavior was first established as thermodynamically feasible in S. cerevisiae [66], and then supported by Metabolic Flux Analysis (MFA) [67]. Later, Camarasa et al. [68] experimentally confirmed it, using NMR isotopic filiation. Hence, the genes involved in succinate dehydrogenase complex and succinyl-CoA ligase were bound to zero, thus forcing the TCA cycle to act as two branches. Also, quinone mediated reactions involving $\mathrm{FADH}_{2}$ and $\mathrm{NADH}$ reoxidation were turned off. Finally, ergosterol, lanosterol and zymosterol upper bound constraints, UB, were set to infinity (assumed to be non-limiting) in anaerobic conditions [69-71].

Furthermore, to ensure the presence of inorganic compounds in every condition studied, phosphate and sulphate concentration in the medium were also set to infinity (UB assumed to be non-limiting).

\section{Dynamic constraints}

Sugar transport is the main rate-limiting step in N-limited fermentations [72]. Known regulatory effects have been previously used by our group to include sugar uptake expressions into a DFBA model [1]. The efficiency of the uptake of sugars is altered by the gene expression, where a set of specific hexose transporters $(\mathrm{HXT})$ are expressed in response to environmental signals $[73,74]$. Moreover, these transporters show competitive inhibition between glucose and fructose, and their activity is modulated by temperature through changes in the activation energy of the process as well as by noncompetitive inhibition by ethanol [75]. Kinetic expressions that considered all these effects were incorporated as UB (upper bound) into the idFV715 model (Additional file 2: eqs. 1, 2).

An empirical function was developed to describe total YAN (Yeast Assimilable Nitrogen) consumption. We incorporated normalized time-varying fluxes for each nitrogen-compound (amino acids/ammonia) into a previously reported equation for total nitrogen consumption $[15,47]$, which was derived from an experimental lookuptable using TableCurve 2D software (Systat Software Inc.). The uptake flux of each $\mathrm{N}$-compound changes according to total nitrogen concentration in the medium, representing a competition for the limited number of nitrogen transporters. These fluxes were included as UB constraints in the LP (Additional files 2: eq. 3).

Yeast faces changing conditions during fermentation, triggering metabolic stress responses and increasing ATP consumption by maintenance $\left(\mathrm{m}_{\mathrm{ATP}}\right)[34,76,77]$, as determined in media with ethanol concentrations above $4 \% \mathrm{v} / \mathrm{v}$ [78]. High temperatures also result in increased cellular maintenance, to restore the membrane potential lost by ion diffusion and from altered protein synthesis $[79,80]$. In addition, sluggish fermentations arising from nitrogen deficiency show higher maintenance costs than normal fermentations [81]. Therefore, to adequately model yeast response to stress, a maintenance term was included that estimate the specific energy requirements to maintain cellular function during fermentation. A lower bound to $\mathrm{m}_{\mathrm{ATP}}$ consumption is set by an empirical function that assigns maintenance costs according to fermentation conditions [47] (Additional file 2: eq. 4). This function, fitted from an experimental lookup-table using TableCurve 2D software, represents the influence of ethanol, high temperature and other energy expenditures associated with sluggish cultures [15].

Accurate simulation of the growth rate in FBA models requires a careful formulation of the biomass equation [38-40,82]. This should reflect observations that biomass composition changes with environmental conditions and as fermentation progresses $[39,81,82]$. Hence, we used a biomass equation that considered new data and experimentally observed variations in the biomass composition of $S$. cerevisiae under different growth conditions. This medium-specific biomass equation was reported in iIN800 model [39] (Additional file 2). Our approach incorporates an empirical carbohydrates accumulation expression (Additional file 2: eq. 5)[15] to the mediumspecific biomass equation [39] to give time-dependent behavior. The equation describing the accumulation of carbohydrates was determined by fitting measured rates to sugar consumption [15]. This function defines an UB in the LP. This final equation is referred to in this article as time-medium-specific biomass equation.

\section{Model validation \\ Experimental fermentations}

idFV715 was validated with data obtained in the laboratory as well as from industrial fermentations. Laboratory data comprised 12 fermentations carried out by our group $[15,79]$ and 8 fermentations provided by the Sciences pour l'Oenologie Research Unit, INRA, Montpellier, France (Drs. Sylvie Dequin and Carole Camarasa, unpublished). Both datasets correspond to alcoholic fermentations performed at several temperatures (20-34 ${ }^{\circ}$ C) under different initial carbon (150-308 g sugar/L) and nitrogen (50-538 $\mathrm{mg} \mathrm{N} / \mathrm{L}$ ) concentrations [15,79]. All were carried out in $1 \mathrm{~L}$ bioreactors using $S$. cerevisiae EC1118 or V5 wine strains.

Industrial data come from 10 anisothermic wine fermentations with different initial sugar concentrations (181-250 g/L) [79]. These were performed during the 2003 vintage in Chile; in fermentation tanks of 40 to 60 $\mathrm{m}^{3}$ of Cabernet Sauvignon inoculated with $S$. cerevisiae EC1118 strain. 


\section{In silico genetic modifications}

The impact of the gene modifications on simulations of batch fermentations, as measured in final product concentrations, was compared with literature data. To simulate the effect of a single gene deletion, the fluxes through the corresponding reactions were constrained to zero during the whole fermentation $[13,42]$. In silico detrimental gene deletions lead to lower growth rates compared to wild-type simulations, yielding lower biomass and/or longer fermentation times.

We assessed the predictive performance of the idFV715 model by comparing simulation results with 35 different environmental and genetic modifications reported in the literature. These include overexpressions [5,83-85], deletions [5,83,84,86-89], insertions [90] and cofactor engineering [5] in single, double and triple mutant strains (Additional file 3 ). The "R" score was used to assess model performance:

$$
R=\frac{D_{i, M, G M}-D_{i, M, W T}}{D_{i, M, W T}}-\frac{D_{i, E, G M}-D_{i, E, W T}}{D_{i, E, W T}}
$$

This expression compares experiments and simulations in terms of the relative changes in the final concentration of given metabolites. Here, $\mathrm{D}$ is the modeling (M) or experimental (E) concentration of the metabolite "i" when it is genetically modified (GM) or wild type (WT). Hence, values of $\mathrm{R}$ close to zero mean good model predictions.

Fermentation conditions that were not given in the literature were estimated to reproduce the respective experimental results as closely as possible. For example, environmental conditions such as temperature, oxygen, sugars, amino acids, ammonia, ethanol and glycerol, were normally given in the literature, but vitamins and inorganic compounds of the medium were not. In addition, deletions and insertions were well described in the literature references; however, the levels of overexpression were not given. Therefore, due to the nature and diversity of information sources, estimating fermentation conditions was often difficult and an additional source of error.

\section{Results and discussion}

\section{Expanding DFBA to genome-scale}

In this study, we expanded a small and reliable 39-equation DFBA model of S. cerevisiae metabolism [15,47] to genome-scale. Our aim was to obtain increased insight into yeast metabolism (given by the genome-scale), while maintaining simulation performance of the previous DFBA model. Therefore, we built a GS-DFBA (idFV715) that accurately predicts the synthesis and consumption profiles of yeast metabolites during the alcoholic fermentation.
To assess the new model, first we analyzed the impact on simulation performance of key model improvements. Then, we validated model results with experimental data.

\section{Assessment of model improvements}

Proper choice of the biomass equation is normally considered a critical component in FBA-based modeling [38-40,82], although adequate handling of ATP maintenance and anaerobic conditions are also key issues that have been overlooked so far. In this study, we explore the impact on the idFV715 model's predictions of maintenance, biomass and anaerobic constraints.

Maintenance Fermentations were simulated anaerobically in N-limiting medium; the variables were low and high temperatures, as well as sluggish and normal conditions. In each case, four maintenance models were assessed: i) mATP constrained according to eq. (4) (Additional file 2), ii) mATP constrained to zero, iii) unconstrained mATP, and iv) mATP constrained to a typical value used in previous models (see Table 1 ).

The most significant difference among model simulations was glycerol prediction. When maintenance flux is constrained according to our maintenance equation (Additional file 2: eq. 4), glycerol predictions are much closer to measured values than the other simulations. In addition, simulations with unbounded or zero maintenance tend to overpredict biomass concentrations, partially explaining the impaired results on glycerol synthesis obtained in these simulations. This is probably due to new environmental conditions where maximization of the growth rate resulted in minimizing ATP costs from glycerol synthesis. Furthermore, when simulations were constrained to a commonly used value from previous genome-scale models (i.e. $1 \mathrm{mmol} / \mathrm{g} \mathrm{DW}$ $\mathrm{h}^{-1}$ ) $[18,63]$, the ATP maintenance expenditure is fixed with a higher value than the output of the maintenance equation proposed in this paper. This higher maintenance cost results in lower biomass and glycerol synthesis and hence in longer fermentation times.

Ethanol prediction also showed significant differences among models. A decrease in glycerol synthesis results in increased carbon fluxes through glycolysis. Hence, unbounded, zero maintenance and constrained-to-previous-value simulations result in higher ethanol contents than those obtained with our maintenance equation (Additional file 2: eq. 4).

Moreover, the latter predicts ethanol concentrations closer to experimental data. The model with our maintenance equation tends to overestimate ethanol synthesis in the nitrogen-lean condition though. To grow under stress, yeasts might divert the carbon flux to other metabolic pathways not accounted for in our model, such as synthesis of lipids and cell-wall 
Table 1 Assessment of maintenance term used in idFV715

\begin{tabular}{|c|c|c|c|c|c|c|c|}
\hline \multicolumn{3}{|c|}{ Initial conditions } & & \multirow[b]{2}{*}{$\begin{array}{c}\text { Time } \\
(\mathrm{H})\end{array}$} & \multirow[b]{2}{*}{$\begin{array}{l}\text { Biomass }(g / \\
\mathrm{L})\end{array}$} & \multirow[b]{2}{*}{$\begin{array}{l}\text { Glycerol (g/ } \\
\text { L) }\end{array}$} & \multirow[b]{2}{*}{$\begin{array}{l}\text { Ethanol }(g / \\
\text { L) }\end{array}$} \\
\hline $\begin{array}{l}\text { Temperature }\left[^{\circ}\right. \\
\mathrm{C}]\end{array}$ & $\underset{\mathrm{L}]}{\text { Nitrogen }[\mathrm{mg} /}$ & $\underset{L]}{\text { Sugars }[g /}$ & & & & & \\
\hline & & & Experimental wt & 455 & 4.65 & ND & ND \\
\hline & & & Model with maintenance & 422 & 4.97 & - & - \\
\hline \multirow[t]{5}{*}{12} & 300 & 268 & Model maintenance constrained to zero & 415 & 5.00 & - & - \\
\hline & & & Model maintenance unbound & 451 & 5.00 & - & - \\
\hline & & & $\begin{array}{l}\text { idFV715 Model using iFF708 maintenance } \\
\text { term }\end{array}$ & 489 & 4.79 & - & - \\
\hline & & & Experimental wt & 700 & $1.49 \pm 0.47$ & $10.98 \pm 0.28$ & $75.41 \pm 6.50$ \\
\hline & & & Model with maintenance & 710 & 0.93 & 9.95 & 96.85 \\
\hline \multirow[t]{5}{*}{28} & 50 & 238 & Model maintenance constrained to zero & 683 & 0.97 & 1.18 & 99.84 \\
\hline & & & Model maintenance unbound & 748 & 0.97 & 1.24 & 117.24 \\
\hline & & & $\begin{array}{l}\text { idFV715 Model using iFF708 maintenance } \\
\text { term }\end{array}$ & 784 & 0.92 & 1.71 & 118.90 \\
\hline & & & Experimental wt & 122 & $5.38 \pm 0.43$ & $7.93 \pm 0.28$ & $107 \pm 3.52$ \\
\hline & & & Model with maintenance & 131 & 5.14 & 10.02 & 106.54 \\
\hline \multirow[t]{3}{*}{28} & 300 & 233 & Model maintenance constrained to zero & 134 & 5.19 & 4.37 & 113.23 \\
\hline & & & Model maintenance unbound & 132 & 5.19 & 15.57 & 106.73 \\
\hline & & & $\begin{array}{l}\text { idFV715 Model using iFF708 maintenance } \\
\text { term }\end{array}$ & 133 & 5.12 & 3.47 & 112.60 \\
\hline
\end{tabular}

Simulations assessing the cost of maintenance in three different environmental conditions. ND means no determined product concentration. Each experiment represents average values from three independent replicates, except for low-temperature conditions.

precursors. Therefore, a careful examination of these components could be useful to improve model results.

Biomass composition Since biomass composition varies throughout fermentation and depends on media composition, a time-medium-specific biomass expression was included (see methods and Additional file 2). To assess the impact on model predictions of this biomass expression, two simulations were carried out using i) current biomass expression; and ii) iFF708 biomass expression (Table 2).

The updated time-dependent biomass expression developed in this work significantly improved biomass predictions compared to simulations using the iFF708 biomass expression. In the first case, using experimental data, an average error ranging from 0.2-0.6 g/L was found. In turn, iFF708 biomass calculations with the GS-DFBA showed deviations between 0.7-1.4 g/L. In both cases, better performances were accomplished in fermentations with standard nitrogen levels $(300 \mathrm{mg} / \mathrm{L}$ YAN), compared to lean-nitrogen cultures. Underestimation of cell growth under low-nitrogen conditions strongly suggests that cells maximize the use of nitrogen compounds under nutrient limitation, a fact that is not accounted in idFV715.

Anaerobic conditions To assess the impact of anaerobic constraints on model predictions, we performed simulations by varying the activation or inhibition of the following specific constraints: i) oxygen uptake constrained

Table 2 Assessment of biomass expression used in idFV715

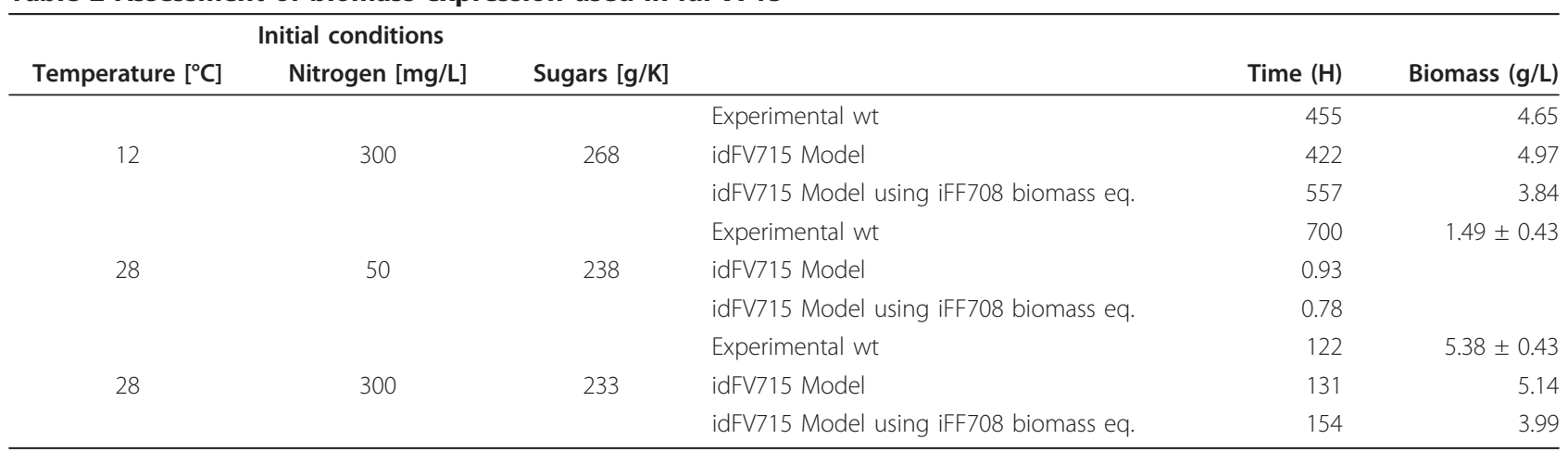

Assessing the impact of different biomass expressions in three different environmental conditions. The "idFV715 model" uses the updated time-medium-specific biomass expression. The "idFV715 model using iFF708" includes the biomass expression of iFF708. Each experiment represents average values from three independent replicates, except for low-temperature conditions. 
to zero, ii) unconstrained sterol and unsatured fatty acids uptake, iii) TCA cycle split up into two branches, and iv) quinone-mediated reactions constrained to zero (Table 3).

Simulations in the absence of oxygen were only feasible when the availability of unsaturated fatty acids and sterols is not constrained (Table 3: model 6). Since $S$. cerevisiae cannot synthesize these compounds (ergosterol and zymosterol) under anaerobic conditions [69-71], this constraint is usually considered in anaerobic models $[29,30]$.

However, restrictions to TCA and quinone-reactions have received little attention and few studies have incorporated transcriptional regulatory information to respond to these environmental conditions [91]. We found that TCA constraints are essential to describe the two separate branches operating under anaerobic conditions - the "oxidative branch" and the "reductive branch" [66-68], which are critical to get accurate predictions. In this condition, glycerol and ethanol synthesis compared very well with experimental data (Table 3: models 1,3 and 4). When the TCA cycle is unconstrained, glycerol synthesis decreases and ethanol increases. This is probably due to the TCA cycle working as a reducing cycle under these unconstrained conditions, reducing the excess NADH. Consequently, glycerol synthesis would be no longer needed, increasing carbon flux through glycolysis and so, ethanol synthesis.

In addition, results showed that quinone-mediated oxidations of $\mathrm{FADH}_{2}$ and NADH play a fundamental role in aerobiosis (not shown); however, under anaerobiosis their effect worsens model predictions. When these reactions are turned off, predictions of fermentation time, biomass, glycerol, and ethanol synthesis are closer to measured values (Table 3: models 2, 4). This is because in idFV715, quinone-mediated reactions do not require oxygen as a precursor, since they always act as electron acceptors, replacing the redox function of glycerol synthesis. Consequently, the rate of ethanol synthesis increases due to a higher carbon flux in glycolysis.

In summary, a significant improvement in glycerol, ethanol, biomass and fermentation time prediction were achieved when the model included the full set of anaerobic constraints described above, in addition to the new maintenance and biomass equations.

\section{Model validation}

We considered for validation 2 types of published experimental data: i) batch fermentation kinetics; and ii) final concentration of metabolic products under genetic/ environmental disturbances. These analyses highlight the differences with our previous model while maintaining model performances.

Fermentation profiles Two sets of batch fermentations with $w t$ yeasts were used. The first set corresponded to 20 laboratory fermentations under different temperature and initial concentrations of nitrogen and sugars. Ten industrial wine fermentations with different initial glucose concentrations and a time varying temperature profile comprised the second set.

Table 4 summarizes performance results for idFV715 validation. The numbers in the respective boxes correspond to the correlation, $\mathrm{R}$, between simulation and experimental data for the whole fermentation process. Figure 2 shows consumption and production profiles for main metabolites and nutrients included in Table 4. Sugar prediction at industrial and laboratory scale shows good agreement with experimental data at several initial sugar conditions (Figure $2 \mathrm{C}, \mathrm{D}$ ). Furthermore, sugar prediction shows good correlation at high-nitrogen and low-nitrogen conditions, reflecting standard and sluggish fermentations (Figure 2E). Therefore, for laboratory and industrial fermentations, idFV715 shows a high degree

Table 3 Assessment of anaerobic constraints used in idFV715

\begin{tabular}{|c|c|c|c|c|c|c|c|c|}
\hline & $\begin{array}{l}\text { Oxygen } \\
\text { uptake }\end{array}$ & $\begin{array}{c}\text { Sterols } \\
\text { uptake** }\end{array}$ & $\begin{array}{l}\text { Quinone } \\
\text { reactions }\end{array}$ & $\begin{array}{l}\text { Complete TCA } \\
\text { cycle* }^{*}\end{array}$ & $\begin{array}{c}\text { Time } \\
(\mathrm{H})\end{array}$ & $\begin{array}{c}\text { Biomass } \\
(\mathrm{g} / \mathrm{L})\end{array}$ & $\begin{array}{l}\text { Glycerol } \\
\text { (g/L) }\end{array}$ & $\begin{array}{l}\text { Ethanol } \\
\text { (g/L) }\end{array}$ \\
\hline Experimental wt & - & - & - & - & 122.0 & $\begin{array}{c}5.38 \pm \\
0.43\end{array}$ & $\begin{array}{c}7.93 \pm \\
0.282\end{array}$ & $\begin{array}{c}107 \pm \\
3.52\end{array}$ \\
\hline $\begin{array}{l}\text { Model } 1 \text { (Used as } \\
\text { wt) }\end{array}$ & off & unlimited & off & off & 130.50 & 5.14 & 10.02 & 106.54 \\
\hline Model 2 & off & unlimited & on & off & 137.50 & 5.10 & 1.50 & 115.07 \\
\hline Model 3 & off & unlimited & off & on & 129.50 & 5.50 & 1.66 & 115.21 \\
\hline Model 4 & off & unlimited & on & on & 137.50 & 5.09 & 2.14 & 114.35 \\
\hline Model 5 & on & unlimited & off & off & 111.50 & 5.49 & 29.44 & 90.44 \\
\hline Model 6 & off & limited & off & off & $L$ & $L$ & $L$ & $L$ \\
\hline
\end{tabular}

Assessing the impact of different factors in anaerobic metabolism. Fermentations were carried out in triplicate in the nitrogen-limited medium $\mathrm{MS} 300$ at $28^{\circ} \mathrm{C}$ Different models represent simulations by varying the activation or inhibition of the specific constraints. $\mathrm{L}=$ lethal; ${ }^{*}$ Complete $\mathrm{TCA}$ cycle means no branches. **Sterols uptake means ergosterol and zymosterol uptake.

$\mathrm{L}=$ lethal

*Complete TCA cycle means no branches

* Sterols uptake mean ergosterol and zymosterol uptake 
Table 4 Fermentation profiles prediction of idFV715

\begin{tabular}{cccccccc}
\hline & Initial Conditions & & \multicolumn{2}{c}{ Lab fermentations } & \multicolumn{2}{c}{ Industrial fermentations } \\
\multicolumn{1}{c}{ Nitrogen } & Sugar & Sugar uptake & Nitrogen uptake & Ethanol & Glycerol & Biomass & Sugar uptake \\
\hline \multirow{2}{*}{ Low 50-200 mg/L } & Low 100-200 g/L & 99.5 & 98.0 & 99.4 & 97.7 & & \\
& High 201-350 g/L & 99.7 & & 99.5 & 95.4 & 84.1 & 97.9 \\
\multirow{2}{*}{ High 201-540 mg/L L } & Low 100-200 g/L & 98.9 & 98.0 & 98.6 & 93.0 & 95.1 & 98.8 \\
& High 201-350 g/L & 99.3 & 99.0 & 99.7 & 98.4 & 95.1 & \\
\hline
\end{tabular}

Fermentation profiles prediction of idFV715 under different medium conditions. Twenty laboratory and ten industrial fermentations, with approx. 25 samples per fermentation, were analyzed. Performance is expressed as percentage of correlation between experimental and model data.

of confidence in sugar uptake profile predictions (glucose and fructose). This step ensures the correct income of energy and carbon to the modeled cell.

Nitrogen is usually the limiting nutrient in alcoholic fermentations and it is linked to the redox-balance that eventually determines the synthesis of glycerol, ethanol and acetate, among other metabolites. Despite its relevance, most current models simply define ammonia uptake as the sole nitrogen source or leave amino acid uptake unbounded; only one bacterial GS-DFBA model applied kinetic parameters to define amino acid uptake, although it has not been experimentally validated [53]. Here, we showed that idFV715 with these new nitrogen features accurately predicts nitrogen uptake experimental profiles, i.e. ammonia and amino acids kinetics, at several initial nitrogen conditions (see Figure 2A, B and Table 4). This feature is useful to define the correct synthesis of redox-linked metabolites.

Simulated profiles of ethanol and glycerol in laboratoryscale fermentations compare well with experimental data (Figure 2G, H). Moreover, prediction of the biomass profile is in relatively close agreement with experimental data; however they are less accurate at low nitrogen conditions (Figure 2F). This could be partly explained by an experimentally observed delay in biomass synthesis in relation to nitrogen uptake that was not captured by idFV715, and also by a lower biomass prediction at these conditions (See section Assessment of model improvements).

Remarkably, when same data sets were used to compare the idFV715 model to our previous reduced DFBA model [15], on average, performances were quite similar (differences in correlation $<5 \%$ ) (Additional file 3). Both models show high correlation and a high predictive ability (less than $5 \%$ of the real value, with $95 \%$ of confidence). Hence, good performance was quite maintained after expansion to the genome-scale.

In summary, despite some differences between model and experimental data, idFV715 predicts relatively well the consumption and synthesis profiles of the main metabolites/nutrients involved in an alcoholic fermentation.

Final product concentration In order to analyze the additional features of the GS-DFBA model, final measured concentrations of metabolic products were compared to idFV715 predictions for 35 fermentations using yeast mutants under a variety of environmental conditions. This set of experiments comprises deletions, overexpressions and insertions in up to three genes (Additional file 3, section 2). These features represent one-step forward compared to our previous model capabilities [15], which had not enough reactions to simulate genetic disturbances.

Analyses of final product concentrations showed that ethanol, glycerol and biomass content - the major carbon compounds in alcoholic fermentation - were predicted in close agreement with experimental data (Figure 3). Indeed, ethanol predictions were accurate, with maximum deviations smaller than 5\%. Glycerol and biomass synthesis showed an acceptable level of confidence with errors smaller than 50\%. In turn, the model often overestimated acetate production. A sensitivity analysis showed that acetate synthesis in the model is highly sensitive to temperature, nitrogen and sugar (Additional file 1). Hence, small errors in these variables (taken from the respective paper or database) can cause large variations in the predicted final acetate concentration. If, in addition, we consider that acetate synthesis represents less than $0.5 \%$ of the initial sugar, it is not surprising that idFV715 does not perform well for acetate prediction. Moreover, synthesis and regulation of acetate are not well known, and FBA models include only carbon and redox governed metabolic rearrangements; transcriptional and protein regulations are not taken into account. In a future version of the model, inclusion of some regulatory network could improve these results as other studies have explored $[46,91,92]$.

Instead of predicting exact final concentration values, the model is able to reproduce the relative effects and trend of given genetic engineering strategies on final metabolites concentrations. For example, our model reproduced the experimental results of Guadalupe et al. (2010) [90]. These authors expressed the E. coli MHPF gene in $S$. cerevisiae, encoding the NAD-dependent acetaldehyde dehydrogenase to restore redox balance and allow the anaerobic growth of a $S$. cerevisiae $\Delta \mathrm{gpd} 1 /$ gpd2 strain through acetate consumption. Including these gene modifications, our model predicted an 

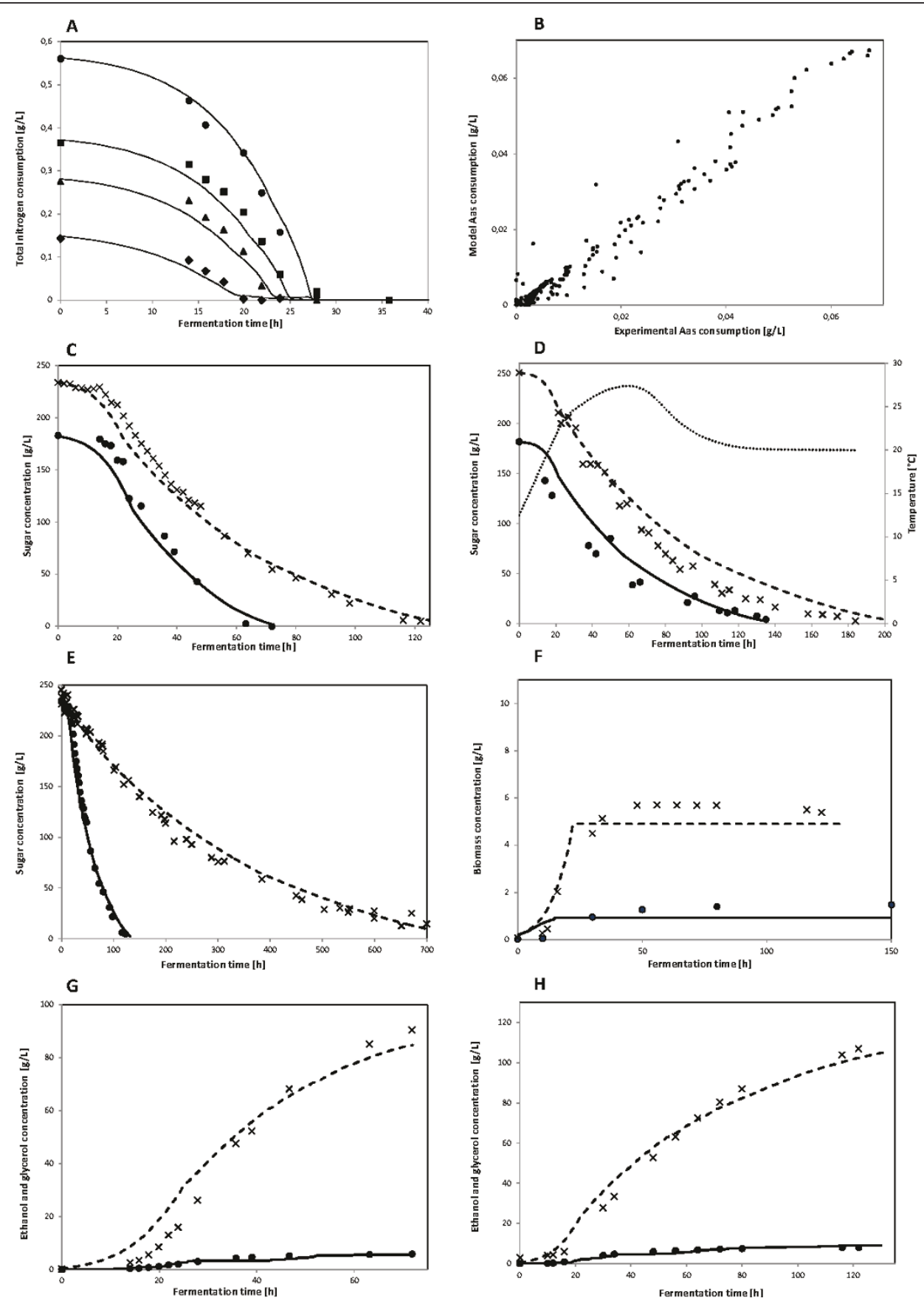

Figure 2 idFV715 model performance. Model predictions of consumption and production rates of the main metabolites and nutrients involved in an alcoholic fermentation. In this figure, symbols represent the experimental data and lines represent model prediction. Measured values were in triplicate with a CV <5\%: A: Experiments and simulations of isothermal, laboratory-scale fermentations, in 4 conditions of totalassimilable-nitrogen $(100,200,300,400$ mg/L of YAN); B: Experiments and simulations of isothermal, laboratory-scale, high-nitrogen fermentations (300 mg/L of YAN, R = 99\%, 400 points), $28^{\circ} \mathrm{C}$; C: Experiments and simulations of isothermal, laboratory-scale, high-nitrogen fermentations. Residual sugar concentration at two representative initial conditions of sugar content in the medium (240 and $182 \mathrm{~g} / \mathrm{L}, 300 \mathrm{mg} / \mathrm{L} \mathrm{YAN}, 28^{\circ} \mathrm{C}$ ); D: Experiments and simulations of anisothermal, industrial-scale, high-nitrogen ( $240 \mathrm{mg} / \mathrm{L}$ YAN) fermentations. The fastest and slowest fermentations are shown. Simulations werefile run assuming a typical temperature profile (dotted-line); E: Isothermal, laboratory-scale, residual sugar concentration for the fastest and the slowest fermentation analyzed, corresponding to $50 \mathrm{mg} / \mathrm{L}$ YAN and $300 \mathrm{mg} / \mathrm{L}$, respectively; F: Isothermal, laboratory-scale, predicted concentration of biomass under $50 \mathrm{mg} / \mathrm{L}$ (closed circles) YAN and $300 \mathrm{mg} / \mathrm{L}(\mathrm{X})$ YAN $28^{\circ} \mathrm{C}$; G: Isothermal, laboratoryscale, predicted concentration of ethanol $(x)$ and glycerol (closed circles) under $240 \mathrm{~g} / \mathrm{L}$ sugar, $300 \mathrm{mg} / \mathrm{L} \mathrm{YAN,} 28^{\circ} \mathrm{C}$; H: Isothermal, laboratoryscale, predicted concentration of ethanol $(X)$ and glycerol (closed circles) under $182 \mathrm{~g} / \mathrm{L}$ sugar, $300 \mathrm{mg} / \mathrm{L} \mathrm{YAN,} 28^{\circ} \mathrm{C}$. 


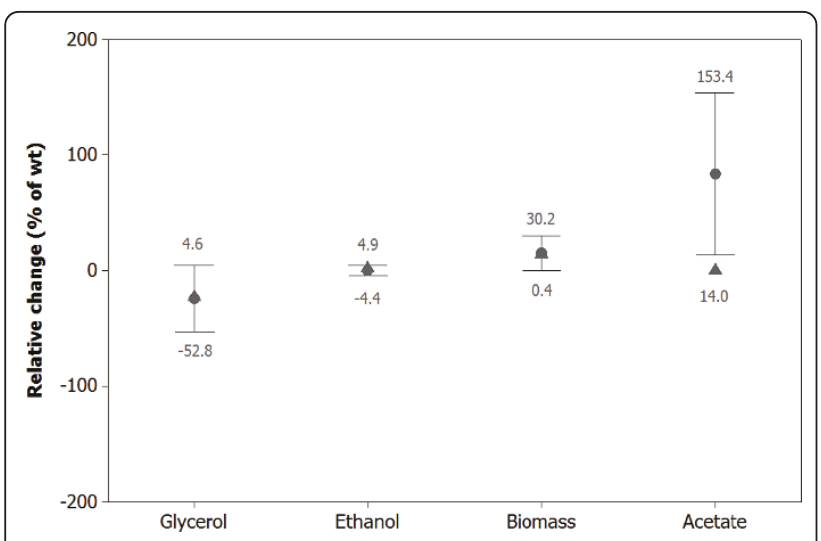

Figure 3 Prediction error distribution for idFV715. Prediction error distribution for 35 different yeast fermentations. The errors compare the relative change between model performance and experimental results, under different environmental conditions (considering $95 \%$ confidence). Mean (•)and median value of each set $(\mathbf{\Delta})$.

increment of ethanol production of 5\%, no glycerol production and consumption of acetate instead of production. Although the model predicts the right trend, the experimental values obtained by Guadalupe et al. (2010) regarding ethanol and acetate were different; they obtained twice the amount of ethanol and almost 7 times more acetate was consumed.

\section{Conclusions}

In this paper, we described the first experimentally validated GS-DFBA model for alcoholic fermentations. Model validation included fermentation profiles and final concentration of fermentation products under different environmental and/or genetic disturbances. We implemented a set of constraints that resulted in key improvements in the physiological response of S. cerevisiae under anaerobic fermentation. Here, we showed that not only biomass expression is a critical component of FBA-based modeling but also ATP maintenance and anaerobic constraints. Furthermore, it is noteworthy that a detailed description of nitrogen-compounds uptake helps to improve results. Therefore, under the conditions studied here, idFV715 closely agrees with final concentrations as well as fermentation profiles of the main metabolites involved in alcoholic fermentation, especially ethanol, glycerol and biomass. Minor compounds such as acetate are not well predicted though, mainly due to unaccounted genetic regulations and model sensitivity. A careful examination in the definition of lipids and cellwall components could help to improve results.

Consequently, idFV715 could be useful to predict the evolution and concentration of the main metabolites from $S$. cerevisiae under different environmental conditions as well as different genetic backgrounds. Given the close predictions of idFV715 regarding ethanol synthesis, this model is especially suitable to design yeast strains for optimum ethanol management in batch fermentations. This model and its future improvements can be used to design new metabolic engineering strategies to optimize fermentation, as well as product synthesis.

\section{Additional material}

Additional file 1: Sensitivity analyses of the idFV715 model. This file includes sensitivity analyses of the effect of time step integration and acetate production in the idFV715 model.

Additional file 2: Kinetic expression used in the idFV715 model. This file includes kinetic expression used in the idFV715 model. Sugar, nitrogen and maintenance expressions are detailed.

Additional file 3: Model validation. This file includes tables where results using previous DFBA model can be directly compared to current model results of Table 4. Also, a description of metabolic engineering conditions was included.

\section{Abbreviations}

DFBA: Dynamic Flux Balance Analysis; GSMM: Genome-Scale Metabolic Model; GS-DFBA: Genome-Scale, Dynamic Flux Balance Analysis; MFA: Metabolic Flux Analysis; MoMA: Minimization of Metabolic Adjustments; NMR: Nuclear Magnetic Resonance; LP: Linear Programming; ODE: Ordinary Differential Equation; TCA: Tricarboxylic acid; YAN: Yeast Assimilable Nitrogen.

\section{Acknowledgements}

The authors thank Drs. Sylvie Dequin and Carole Camarasa for providing fermentation data to validate the model. The authors also thank Dr. Jens Nielsen for providing the genome-scale metabolic network. This work was funded by VINNOVA 05CTE01-06. FAV acknowledges support by CONICYT. We also acknowledge the suggestions of the anonymous referees that contributed to significantly improve our manuscript. Finally, we are grateful to Lisa Gingles for editing this manuscript.

\section{Authors' contributions}

The authors FAV, FP, JRP and EA, conceived and designed the experiments. FAV performed the experiments and analyzed the data. All authors have read and approved the final manuscript.

Received: 19 June 2010 Accepted: 19 May 2011 Published: 19 May 2011

\section{References}

1. Kong QX, Cao LM, Zhang AL, Chen X: Overexpressing GLT1 in gpd1 mutant to improve the production of ethanol of Saccharomyces cerevisiae. Appl Microbiol Biotechnol 2007, 73:1382-1386.

2. Kong QX, Gu JG, Cao LM, Zhang AL, Chen X, Zhao XM: Improved production of ethanol by deleting FPS1 and over-expressing GLT1 in Saccharomyces cerevisiae. Biotechnol Lett 2006, 28:2033-2038.

3. Valadi H, Larsson C, Gustafsson L: Improved ethanol production by glycerol-3-phosphate dehydrogenase mutants of Saccharomyces cerevisiae. Appl Microbiol Biotechnol 1998, 50:434-439.

4. Seo HB, Kim SS, Lee HY, Jung KH: High-level production of ethanol during fed-batch ethanol fermentation with a controlled aeration rate and nonsterile glucose powder feeding of Saccharomyces cerevisiae. Biotechnol Biop Eng 2009, 14:591-598.

5. Ehsani M, Fernandez MR, Biosca JA, Julien A, Dequin S: Engineering 2, 3butanediol dehydrogenase to reduce acetoin formation by glycerol overproducing low-alcohol wine yeast. App/ Environ Microbiol 2009, 75:3196-3205. 
6. Hahn-Hägerdal B, Karhumaa K, Fonseca C, Spencer-Martins I, GorwaGrauslund MF: Towards industrial pentose-fermenting yeast strains. Appl Microbiol Biotechnol 2007, 74:937-953.

7. Nevoigt E: Progress in metabolic engineering of Saccharomyces cerevisiae Microbiol Mol Biol Rev 2008, 72.379-412

8. Matsushika A, Inoue H, Kodaki T, Sawayama S: Ethanol production from xylose in engineered Saccharomyces cerevisiae strains: current state and perspectives. Appl Microbiol Biotechnol 2009, 84:37-53.

9. Ra M, Mm D: Simple constrained optimization view of acetate overflow in E. coli. Biotechnol Bioeng 1990, 35:732-738.

10. Ramakrishna R, Edwards JS, McCulloch A, Palsson BO: Flux-balance analysis of mitochondrial energy metabolism: consequences of systemic stoichiometric constraints. Am J Physiol 2001, 280:695-704.

11. Kauffman KJ, Prakash P, Edwards JS: Advances in flux balance analysis. Curr Opin Biotechnol 2003, 14:491-496

12. Edwards JS, Palsson BO: The Escherichia coli MG1655 in silico metabolic genotype: its definition, characteristics, and capabilities. Proc Natl Acad Sci USA 2000, 97:5528-5533.

13. Price ND, Papin JA, Schilling CH, Palsson BO: Genome-scale microbial in silico models: the constraints-based approach. Trends Biotechnol 2003, 21:162-169.

14. Segre D, Vitkup D, Church GM: Analysis of optimality in natural and perturbed metabolic networks. Proc Natl Acad Sci USA 2002, 99:15112-15117.

15. Pizarro F, Varela C, Martabit C, Bruno C, Perez-Correa JR, Agosin E: Coupling kinetic expressions and metabolic networks for predicting wine fermentations. Biotechnol Bioeng 2007, 98:986-998.

16. Raghunathan AU, Perez-Correa JR, Agosin E, Biegler LT: Parameter estimation in metabolic flux balance models for batch fermentationFormulation \& Solution using Differential Variational Inequalities (DVIs). Ann Op Res 2006, 148:251-270.

17. Alper HHS: Development of systematic and combinatorial approaches for the metabolic engineering of microorganisms. PhD Thesis MIT, Metabolic engineering department; 2006.

18. Forster J, Famili I, Fu P, Palsson BO, Nielsen J: Genome-scale reconstruction of the Saccharomyces cerevisiae metabolic network. Genome Res 2003, 13:244-253.

19. Duarte NC, Herrgård MJ, Palsson B: Reconstruction and Validation of Saccharomyces cerevisiae iND750, a Fully Compartmentalized GenomeScale Metabolic Model. Genome Res 2004, 14:1298-1309.

20. Pharkya $P$, Burgard AP, Maranas CD: OptStrain: a computational framework for redesign of microbial production systems. Genome Res 2004, 14:2367-2376.

21. Ranganathan S, Suthers PF, Maranas CD: OptForce: An Optimization Procedure for Identifying All Genetic Manipulations Leading to Targeted. PLoS Comp Biol 2010, 6:1-11.

22. Bro C, Regenberg B, Förster J, Nielsen J: In silico aided metabolic engineering of Saccharomyces cerevisiae for improved bioethanol production. Metab Eng 2006, 8:102-111.

23. Alper H, Jin YS, Moxley JF, Stephanopoulos G: Identifying gene targets for the metabolic engineering of lycopene biosynthesis in Escherichia coli. Metab Eng 2005, 7:155-164.

24. Fong SS, Burgard AP, Herring CD, Knight EM, Blattner FR, Maranas CD Palsson BO: In silico design and adaptive evolution of Escherichia coli for production of lactic acid. Biotechnol Bioeng 2005, 91:643-648.

25. Wang Q, Chen X, Yang Y, Zhao X: Genome-scale in silico aided metabolic analysis and flux comparisons of Escherichia coli to improve succinate production. Appl Microbiol Biotechnol 2006, 73:887-894.

26. Burgard AP, Pharkya P, Maranas CD: Optknock: a bilevel programming framework for identifying gene knockout strategies for microbial strain optimization. Biotechnol Bioeng 2003, 84:647-657.

27. Park JH, Lee KH, Kim TY, Lee SY: Metabolic engineering of Escherichia coli for the production of L-valine based on transcriptome analysis and in silico gene knockout simulation. Proc Natl Acad Sci USA 2007, 104:7797-7802.

28. Lee KH, Park JH, Kim TY, Kim HU, Lee SY: Systems metabolic engineering of Escherichia coli for L-threonine production. Mol Syst Biol 2007, 3:1744-4292.

29. Hjersted JL, Henson MA, Mahadevan R: Genome-scale analysis of Saccharomyces cerevisiae metabolism and ethanol production in fedbatch culture. Biotechnol Bioeng 2007, 97:1190-1204.
30. Hjersted $J L$, Henson MA: Steady-state and dynamic flux balance analysis of ethanol production by Saccharomyces cerevisiae. IET Systems Biology 2009, 3:167-179.

31. Dikicioglu D, Pir P, Onsan Zl, Ulgen KO, Kirdar B, Oliver SG: Integration of metabolic modeling and phenotypic data in evaluation and improvement of ethanol production using respiration-deficient mutants of Saccharomyces cerevisiae. Appl Environ Microbiol 2008, 74:5809-5816.

32. Bisson LF: The Biotechnology of Wine Yeast. Food Biotechnol 2004 18:63-96.

33. Pretorius IS: Tailoring wine yeast for the new millennium: novel approaches to the ancient art of winemaking. Yeast 2000, 16:675-729.

34. Pizarro F, Vargas FA, Agosin E: A systems biology perspective of wine fermentations. Yeast 2007, 24:977-991.

35. Pharkya P, Maranas CD: An optimization framework for identifying reaction activation/inhibition or elimination candidates for overproduction in microbial systems. Metab Eng 2006, 8:1-13.

36. Patil KR, Rocha I, Förster J, Nielsen J: Evolutionary programming as a platform for in silico metabolic engineering. BMC Bioinformatics 2005, 6:308-320.

37. Otero JM, Nielsen J: Industrial systems biology. Biotechnol Bioeng 2009, 105:439-460

38. Mo ML, Palsson B, Herrgård MJ: Connecting extracellular metabolomic measurements to intracellular flux states in yeast. BMC Sys Biol 2009, 3:37-54.

39. Nookaew I, Jewett MC, Meechai A, Thammarongtham C, Laoteng K, Cheevadhanarak S, Nielsen J, Bhumiratana S: The genome-scale metabolic model ilN 800 of Saccharomyces cerevisiae and its validation: a scaffold to query lipid metabolism. BMC Sys Biol 2008, 2:71.

40. Kuepfer L, Sauer U, Blank LM: Metabolic functions of duplicate genes in Saccharomyces cerevisiae. Genome Res 2005, 15:1421-1430.

41. Dobson PD, Smallbone K, Jameson D, Simeonidis E, Lanthaler K, Pir P, Lu C, Swainston N, Dunn WB, Fisher P: Further developments towards a genome-scale metabolic model of yeast. BMC Systems Biology 2010, 4:145-151

42. Varma A, Palsson BO: Stoichiometric flux balance models quantitatively predict growth and metabolic by-product secretion in wild-type Escherichia coli W3110. Appl Environ Microbiol 1994, 60:3724-3731.

43. Mahadevan R, Edwards JS, Doyle lii FJ: Dynamic flux balance analysis of diauxic growth in Escherichia coli. Biophys J 2002, 83:1331-1340.

44. Anesiadis N, Cluett WR, Mahadevan R: Dynamic metabolic engineering for increasing bioprocess productivity. Metab Eng 2008, 10:255-266

45. Gaspard L, Joeri B, Jo M, Ellen VH, Wim S, Erick V, Peter A: Dynamic Metabolic Flux Analysis Demonstrated on Cultures Where the Limiting Substrate Is Changed from Carbon to Nitrogen and Vice Versa. J Biomed Biotechnol 2010, 2010:19.

46. Lee JM, Gianchandani EP, Eddy JA, Papin JA: Dynamic analysis of integrated signaling, metabolic, and regulatory networks. PLoS Comput Biol 2008, 4:e1000086.

47. Sainz J, Pizarro F, Perez-Correa J, Agosin E: Modeling of yeast metabolism and process dynamics in batch fermentation. Biotechnol Bioeng 2003, 81:818-828.

48. Luo R, Wei H, Ye L, Wang K, Chen F, Luo L, Liu L, Li Y, Crabbe MJC, Jin L: Photosynthetic metabolism of C3 plants shows highly cooperative regulation under changing environments: A systems biological analysis. Proc Natl Acad Sci USA 2009, 106:847-852.

49. Uygun K, Matthew HWT, Huang Y: DFBA-LQR: An optimal control approach to flux balance analysis. Ind Eng Chem Res 2006, 45:8554-8564.

50. Luo RY, Liao S, Tao GY, Li YY, Zeng S, Li YX, Luo Q: Dynamic analysis of optimality in myocardial energy metabolism under normal and ischemic conditions. Mol Syst Biol 2006, 2:1-6.

51. Salimi F, Zhuang K, Mahadevan R: Genome scale metabolic modeling of a clostridial co culture for consolidated bioprocessing. Biotechnologu 2010, 5:726-738

52. Zhuang K, Izallalen M, Mouser P, Richter H, Risso C, Mahadevan R, Lovley DR: Genome- scale dynamic modeling of the competition between Rhodoferax and Geobacter in anoxic subsurface environments. The ISME Journal.

53. Meadows AL, Karnik R, Lam H, Forestell S, Snedecor B: Application of dynamic flux balance analysis to an industrial Escherichia coli fermentation. Metab Eng 2009, 12:150-160. 
54. Oddone GM, Mills DA, Block DE: A dynamic, genome-scale flux model of Lactococcus lactis to increase specific recombinant protein expression. Metab Eng 2009, 11:367-381.

55. Famili I, Forster J, Nielsen J, Palsson BO: Saccharomyces cerevisiae Phenotypes Can Be Predicted by Using Constraint-Based Analysis of a Genome-Scale Reconstructed Metabolic Network. Proc Natl Acad Sci USA 2003, 100:13134-13139.

56. Edwards JS, Ibarra RU, Palsson BO: In silico predictions of Escherichia col metabolic capabilities are consistent with experimental data. Nat Biotechnol 2001, 19:125-130.

57. Mahadevan $\mathrm{R}$, Schilling $\mathrm{CH}$ : The effects of alternate optimal solutions in constraint-based genome-scale metabolic models. Metab Eng 2003, 5:264-276.

58. ter Linde JJM, Liang H, Davis RW, Steensma HY, van Dijken JP, Pronk JT: Genome-Wide Transcriptional Analysis of Aerobic and Anaerobic Chemostat Cultures of Saccharomyces cerevisiae. J Bacteriol 1999, 181:7409-7413.

59. van den Brink J, Daran-Lapujade P, Pronk JT, de Winde JH: New insights into the Saccharomyces cerevisiae fermentation switch: Dynamic transcriptional response to anaerobicity and glucose-excess. BMC Genomics 2008, 9:100.

60. Boer VM, de Winde JH, Pronk JT, Piper MD: The genome-wide transcriptional responses of Saccharomyces cerevisiae grown on glucose in aerobic chemostat cultures limited for carbon, nitrogen, phosphorus, or sulfur. J Biol Chem 2003, 278:3265-3274.

61. Tai SL, Boer VM, Daran-Lapujade P, Walsh MC, de Winde JH, Daran JM, Pronk JT: Two- dimensional transcriptome analysis in chemostat cultures. Combinatorial effects of oxygen availability and macronutrient limitation in Saccharomyces cerevisiae. J Biol Chem 2005, 280:437-447.

62. Kwast KE, Lai LC, Menda N, James DT, Aref S, Burke PV: Genomic analyses of anaerobically induced genes in Saccharomyces cerevisiae: functional roles of Rox 1 and other factors in mediating the anoxic response. $J$ Bacteriol 2002, 184:250-265.

63. Verduyn C, Postma E, Scheffers WA, Van Dijken JP: Physiology of Saccharomyces cerevisiae in anaerobic glucose-limited chemostat cultures. J Gen Appl Microbiol 1990, 136:395-403.

64. Verduyn C, Stouthamer AH, Scheffers WA, Dijken JP: A theoretical evaluation of growth yields of yeasts. Antonie Van Leeuwenhoek 1991, 59:49-63.

65. Bakker BM, Overkamp KM, van Maris AJ, Kotter P, Luttik MA, van Dijken JP, Pronk JT: Stoichiometry and compartmentation of NADH metabolism in Saccharomyces cerevisiae. FEMS Microbiol Rev 2001, 25:15-37.

66. Lupiañez JA, Machado A, Nuñez de Castro I, Mayor F: Succinic acid production by yeasts grown under different hypoxic conditions. Mol Cell Biochem 1974, 3:113-116.

67. Nissen TL, Schulze U, Nielsen J, Villadsen J: Flux distributions in anaerobic, glucose-limited continuous cultures of Saccharomyces cerevisiae. Microbiology 1997, 143:203-218.

68. Camarasa C, Grivet JP, Dequin S: Investigation by 13C-NMR and tricarboxylic acid (TCA) deletion mutant analysis of pathways for succinate formation in Saccharomyces cerevisiae during anaerobic fermentation. Microbiology 2003, 149:2669-2678.

69. Fornairon-Bonnefond C, Demaretz V, Rosenfeld E, Salmon JM: Oxygen addition and sterol synthesis in Saccharomyces cerevisiae during enological fermentation. J Biosci Bioeng 2002, 93:176-182.

70. Andreasen AA, Stier TJB: Anaerobic nutrition of Saccharomyces cerevisiae. I. Ergosterol requirement for growth in a defined medium. J Cel Comp Physiol 1953, 41:23-26.

71. Andreasen AA, Stier TJB: Anaerobic nutrition of saccharomyces cerevisiae. II. Unsaturated fatty and requirement for growth in a defined medium. $J$ Cel Comp Physiol 1954, 43:271-281.

72. Bisson LF: Stuck and sluggish fermentations. Am J Enol Viticul 1999, 50:107.

73. Perez M, Luyten K, Michel R, Riou C, Blondin B: Analysis of Saccharomyces cerevisiae hexose carrier expression during wine fermentation: both low- and high-affinity Hxt transporters are expressed. FEMS Yeast Res 2005, 5:351-361

74. Reifenberger E, Boles E, Ciriacy M: Kinetic characterization of individual hexose transporters of Saccharomyces cerevisiae and their relation to the triggering mechanisms of glucose repression. Eur J Biochem 1997, 245:324-333.
75. Luyten K, Riou C, Blondin B: The hexose transporters of Saccharomyces cerevisiae play different roles during enological fermentation. Yeast 2002, 19:713-726.

76. Gasch AP, Werner-Washburne M: The genomics of yeast responses to environmental stress and starvation. Funct Integr Genomics 2002, 2:181-192

77. Rossouw D, Bauer FF: Comparing the transcriptomes of wine yeast strains: toward understanding the interaction between environment and transcriptome during fermentation. Appl Microbiol Biotechnol 2009, 84:937-954.

78. Piper PW, Talreja K, Panaretou B, Moradas-Ferreira P, Byrne K, Praekelt UM, Meacock $\mathrm{P}$, Recnacq $\mathrm{M}$, Boucherie $\mathrm{H}$ : Induction of major heat-shock proteins of Saccharomyces cerevisiae, including plasma membrane Hsp30, by ethanol levels above a critical threshold. Microbiology 1994 140:3031-3038.

79. Pizarro FJ, Jewett MC, Nielsen J, Agosin E: Growth temperature exert differential physiological and transcriptional responses in laboratory and wine strains of Saccharomyces cerevisiae. Appl Environ Microbiol 2008, 74:6358-6368.

80. Postmus J, Canelas AB, Bouwman J, Bakker BM, van Gulik W, de Mattos M, Brul S, Smits GJ: Quantitative analysis of the high temperature-induced glycolytic flux increase in Saccharomyces cerevisiae reveals dominant metabolic regulation. J Biol Chem 2008, 283:23524-23532.

81. Varela C, Pizarro F, Agosin E: Biomass content governs fermentation rate in nitrogen- deficient wine musts. Appl Environ Microbiol 2004 70:3392-3400.

82. Pramanik J, Keasling JD: Effect of Escherichia coli biomass composition on central metabolic fluxes predicted by a stoichiometric model. Biotechnol Bioeng 2000, 60:230-238

83. Cambon B, Monteil V, Remize F, Camarasa C, Dequin S: Effects of GPD1 overexpression in Saccharomyces cerevisiae commercial wine yeast strains lacking ALD6 genes. Appl Environ Microbiol 2006, 72:4688-4694.

84. Eglinton JM, Heinrich AJ, Pollnitz AP, Langridge P, Henschke PA, de Barros Lopes M: Decreasing acetic acid accumulation by a glycerol overproducing strain of Saccharomyces cerevisiae by deleting the ALD 6 aldehyde dehydrogenase gene. Yeast 2002, 19:295-301.

85. Remize F, Roustan J, Sablayrolles J, Barre P, Dequin S: Glycerol overproduction by engineered Saccharomyces cerevisiae wine yeast strains leads to substantial changes in by-product formation and to a stimulation of fermentation rate in stationary phase. Appl Environ Microbiol 1999, 65:143-149.

86. Guo Z, Zhang L, Ding Z, Wang ZX, Shi GY: Interruption of glycerol pathway in industrial alcoholic yeasts to improve the ethanol production. Appl Microbiol Biotechnol 2009, 82:287-292

87. Remize F, Andrieu E, Dequin S: Engineering of the pyruvate dehydrogenase bypass in Saccharomyces cerevisiae: role of the cytosolic $\mathrm{Mg} 2+$ and mitochondrial $\mathrm{K}+$ acetaldehyde dehydrogenases Ald6p and Ald4p in acetate formation during alcoholic fermentation. Appl Environ Microbiol 2000, 66:3151-3159.

88. Saint-Prix F, Bonquist $L$, Dequin S: Functional analysis of the ALD gene family of Saccharomyces cerevisiae during anaerobic growth on glucose: the NADP+-dependent Ald6p and Ald5p isoforms play a major role in acetate formation. Microbiology 2004, 150:2209-2220.

89. Zhang A, Kong Q, Cao L, Chen X: Effect of FPS1 deletion on the fermentation properties of Saccharomyces cerevisiae. Lett Appl Microbio 2007, 44:212-217.

90. Guadalupe Medina V, Almering MJH, van Maris AJA, Pronk JT: Elimination of glycerol production in anaerobic cultures of a Saccharomyces cerevisiae strain engineered to use acetic acid as an electron acceptor. Appl Environ Microbiol 2010, 76:190-195.

91. Herrgård MJ, Lee BS, Portnoy V, Palsson B: Integrated analysis of regulatory and metabolic networks reveals novel regulatory mechanisms in Saccharomyces cerevisiae. Genome Res 2006, 16:627-635.

92. Covert MW, Palsson BO: Constraints-based models: regulation of gene expression reduces the steady-state solution space. J Theor Biol 2003, 221:309-325.

doi:10.1186/1752-0509-5-75

Cite this article as: Vargas et al:: Expanding a dynamic flux balance model of yeast fermentation to genome-scale. BMC Systems Biology 2011 5:75. 